\section{Hematoma subcapsular hepático roto en síndrome HELLP}

\author{
MARÍA PAZ HENRÍQUEZ-VILLASECA ${ }^{1}$, \\ ALEJANDRA CATALÁN-BARAHONA², JOSÉ LATTUS-OLMOS², \\ KARINA VARGAS-VALDEBENITO3 ${ }^{3}$, SOLANGE SILVA-RUZ ${ }^{3}$
}

\section{Ruptured subscapular liver hematoma in context of HELLP syndrome.}

Background: Ruptured subscapular liver hematoma in context of HELLP syndrome is a rare complication with high mortality. Clinical presentation is nonspecific: epigastric pain, right upper quadrant pain and shoulder pain, should however provide high index of suspicion. Termination of pregnancy is the only definitive treatment with a beneficial effect on the mother and the fetus. According to the hemodynamic status of the patient, treatment can be conservative through hepatic artery embolization, or surgical management, via contained packing of the bleeding area. Aim: To report seven cases of ruptured subscapular liver hematomas treated in a period of 13 years. Material and Methods: Review of medical records of patients discharged with the diagnosis from 2002 to 2015. Results: We identified seven women aged 30 to 44 years, for a total of 86,858 live births. At presentation, mean gestational age was 36 weeks. Five patients reported epigastric pain and three shoulder pain. All patients met the criteria for HELPP syndrome. All patients were operated on, and packing of the bleeding zone was carried out in six. One patient died. Two newborns died. Conclusions: The features of these patients may help in the management of this uncommon but severe condition.

(Rev Med Chile 2018; 146: 753-761)

Key words: HELLP Syndrome; Pregnancy Complications; Rupture, Spontaneous.

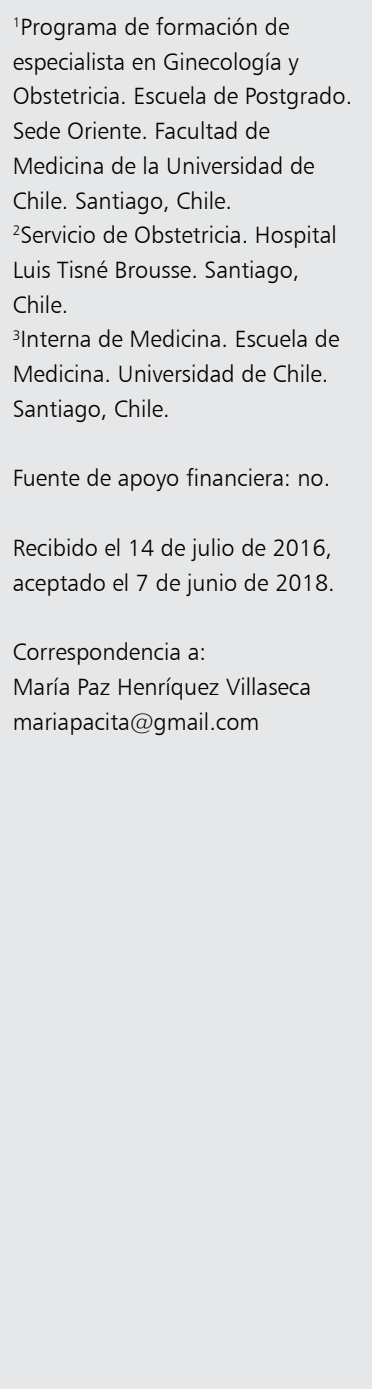

1Programa de formación de Sede Oriente. Facultad de Medicina de la Universidad de Chile. Santiago, Chile. Servicio de Obstetricia. Hospital Chile.

${ }^{3}$ Interna de Medicina. Escuela de Medicina. Universidad de Chile. Santiago, Chile.

Fuente de apoyo financiera: no.

Recibido el 14 de julio de 2016 aceptado el 7 de junio de 2018

Correspondencia a:

María Paz Henríquez Villaseca mariapacita@gmail.com
E 1 síndrome HELLP está caracterizado por hemólisis, enzimas hepáticas elevadas y plaquetopenia, suele asociarse a síndromes hipertensivos del embarazo (SHE). Su incidencia es de $0,1-0,8 \%$ de todos los embarazos y de $10-20 \%$ en mujeres con preeclampsia severa/eclampsia ${ }^{1-3}$. El hematoma subcapsular hepático (HSCH) y su evolución a una rotura hepática $(\mathrm{RH})$ se presenta como una complicación infrectuente del síndrome HELLP, encontrándose una incidencia de $1 / 45.000$ a $1 / 225.000$ embarazos $^{6-7}$, no obstante, posee un importante índice de morbimortalidad materno fetal. La mortalidad por HELLP es de 2-3\%, aumentando a 59\%-62\% ante la presencia de un $\mathrm{HSCH}^{6,13-17}$. Dada la rápida evolución y compromiso vital de este cuadro, es importante contar con elementos que permitan una oportuna sospecha diagnóstica para una acción terapéutica oportuna y pertinente.

En esta serie se muestran los siete casos que se presentaron a lo largo de trece años en nuestro servicio, donde se describe presentación, cuadro clínico y laboratorio, así como la conducta terapéutica realizada y evolución, logrando esta- 
blecer la tasa de mortalidad materna y perinatal en nuestra realidad local. Se hizo una revisión de la literatura enfocada en elementos prácticos que permitan desarrollar una sospecha clínica adecuada, exámenes útiles para la confirmación diagnóstica, diversos manejos disponibles, junto con la descripción de algunas experiencias nacionales como internacionales.

\section{Material y Método}

Se procedió a la revisión de la ficha clínica y epicrisis de las pacientes con diagnóstico de hematoma subcapsular hepático roto asociado a síndrome HELLP tratadas en la Unidad de Cuidados Intensivos (UCI) del Hospital Dr Luis Tisné Brousse, en el período desde enero de 2002 a agosto de 2015, consignando datos demográficos y clínicos.

\section{Resultados}

Durante el período en estudio, se presentaron 7 casos de hematoma subcapsular hepático roto (Tabla 1), para un total de 86.858 nacidos vivos, lo que nos da una incidencia de 1/12.408.

La edad promedio de las pacientes fue de 34 años, con una mediana de 32 años, y un rango entre 30 y 44 años.

Con respecto al momento del diagnóstico del HSCHR, en cinco casos fue durante el embarazo, con una edad gestacional promedio de presentación de 36 semanas, con un rango de $35+1$ a $37+2$ semanas, y en dos caso se presentó en el postparto inmediato (uno en puérpera inmediata de parto prematuro de 35 semanas, espontáneo, y otro en puérpera inmediata de cesárea de urgencia en embarazo de 32 semanas por preeclampsia severa y síndrome HELLP).

En 4 de los 7 casos, el diagnóstico fue intraoperatorio por cesárea $(57,1 \%)$, en 2 casos se realizó el diagnóstico por ecografía abdominal previo la interrupción del embarazo $(28,6 \%)$ y en un caso se realizó por laparotomía media supra e infraumbilical (LMSIU) exploradora (postcesárea de urgencia) (14,3\%). En 3 de los casos hubo compromiso de lóbulo hepático derecho $(42,85 \%)$, en 3 hubo compromiso de ambos lóbulos $(42,85 \%)$ y en solo un caso $(14,28 \%)$ hubo compromiso de lóbulo hepático izquierdo.

\section{Cuadro clínico}

En nuestra revisión se observó que las pacientes con HSCSR presentaron uno o más de los siguientes signos y síntomas:

Epigastralgia fue el síntoma más frecuente, presentándose en $5 / 7(71,4 \%)$, omalgia en $3 / 7$ $(42,8 \%)$, dolor abdominal en hipocondrio derecho en $1 / 7(14,3 \%)$, cervicalgia en $1 / 7(14,3 \%)$ y cefalea en $1 / 7(14,3 \%)$ de las pacientes.

\section{Hallazgos de laboratorio}

En la Tabla 1 se describen los hallazgos bioquímicos de las pacientes que presentaron $\mathrm{HSCH}$ desde su ingreso al Servicio de Urgencias y durante su estancia en el hospital.

Observamos al ingreso que solo 3 de las 7 pacientes presentaron anemia, respecto a las transaminasas, solo una presentó valores en rango normal de transaminasa glutámico pirúvica y levemente aumentada de transaminasa glutámico oxalacética, el resto todas estaban alteradas. La deshidrogenasa láctica $(\mathrm{LDH})$ estaba normal solo en 1 paciente al ingreso, sin embargo, en su evolución igualmente se elevó. El recuento plaquetario se encontró alterado en todas las pacientes.

\section{Preeclampsia y síndrome HELLP}

Seis de las 7 pacientes presentaron el diagnóstico de preeclampsia severa $(85,7 \%)$, y todas cumplieron con los criterios diagnósticos de síndrome HELLP al momento de la rotura del HSCH.

\section{Terapia}

Con respecto al manejo terapéutico del hematoma subcapsular, en todos los casos el manejo fue quirúrgico (Figura 1), el cual consistió en taponamiento con compresas (packing) en 6 de 7 casos, y solo en uno se realizó aseo, aplicación de un hemostático absorbible/(Surgicel $\left.{ }^{\mathrm{R}}\right)$ y refuerzo con malla de vicryl. Solo en un caso se utilizó factor VII recombinante, 4 ampollas, además del manejo quirúrgico.

En promedio, se requirieron 6 unidades de glóbulos rojos, 8 unidades de plasma fresco congelado (PFC), 12 unidades de plaquetas y 10 unidades de crioprecipitados en cada paciente.

En ninguna paciente se realizó ligadura de arteria hepática. No se dispone de embolización arterial en nuestro hospital. 


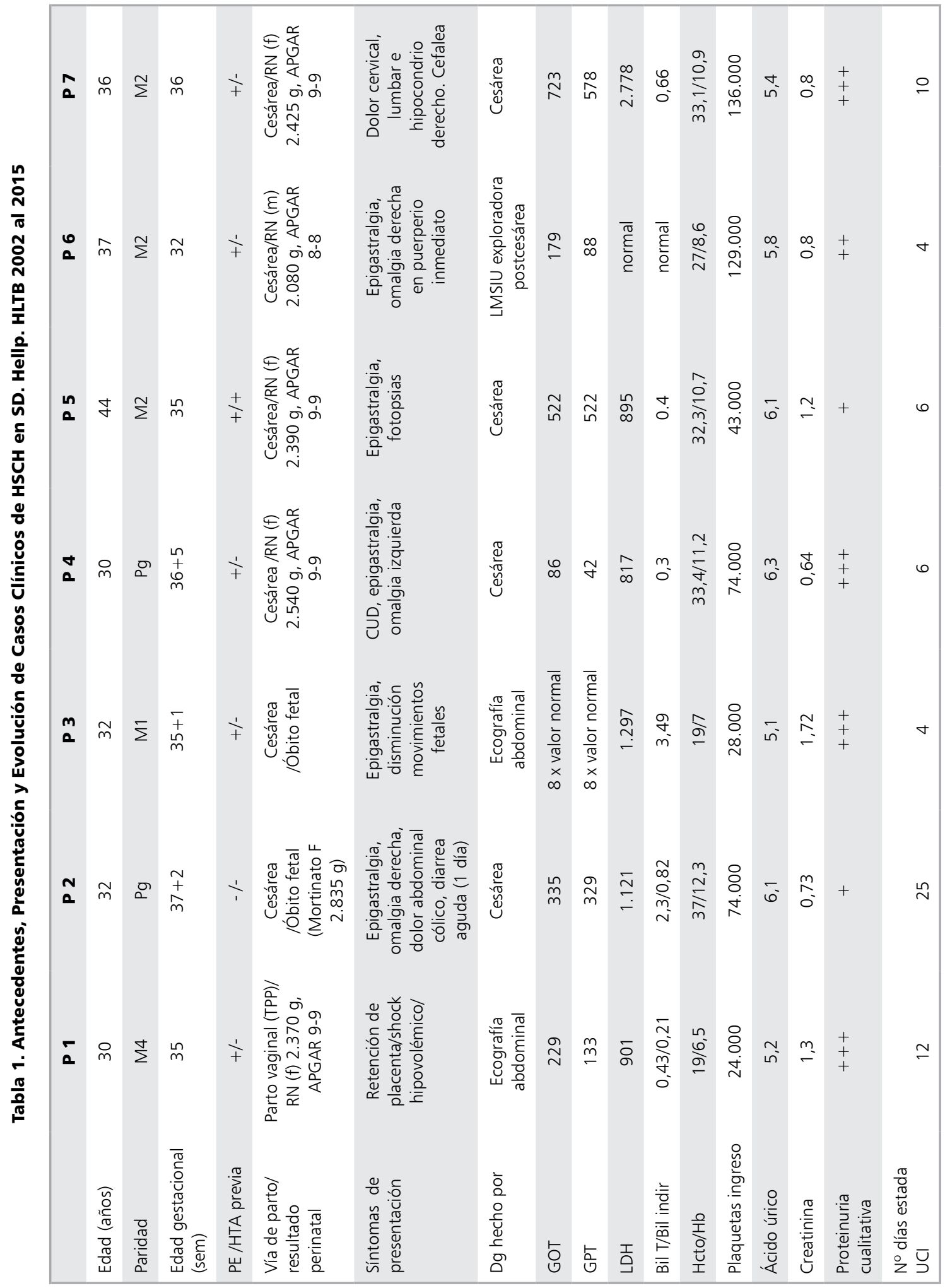




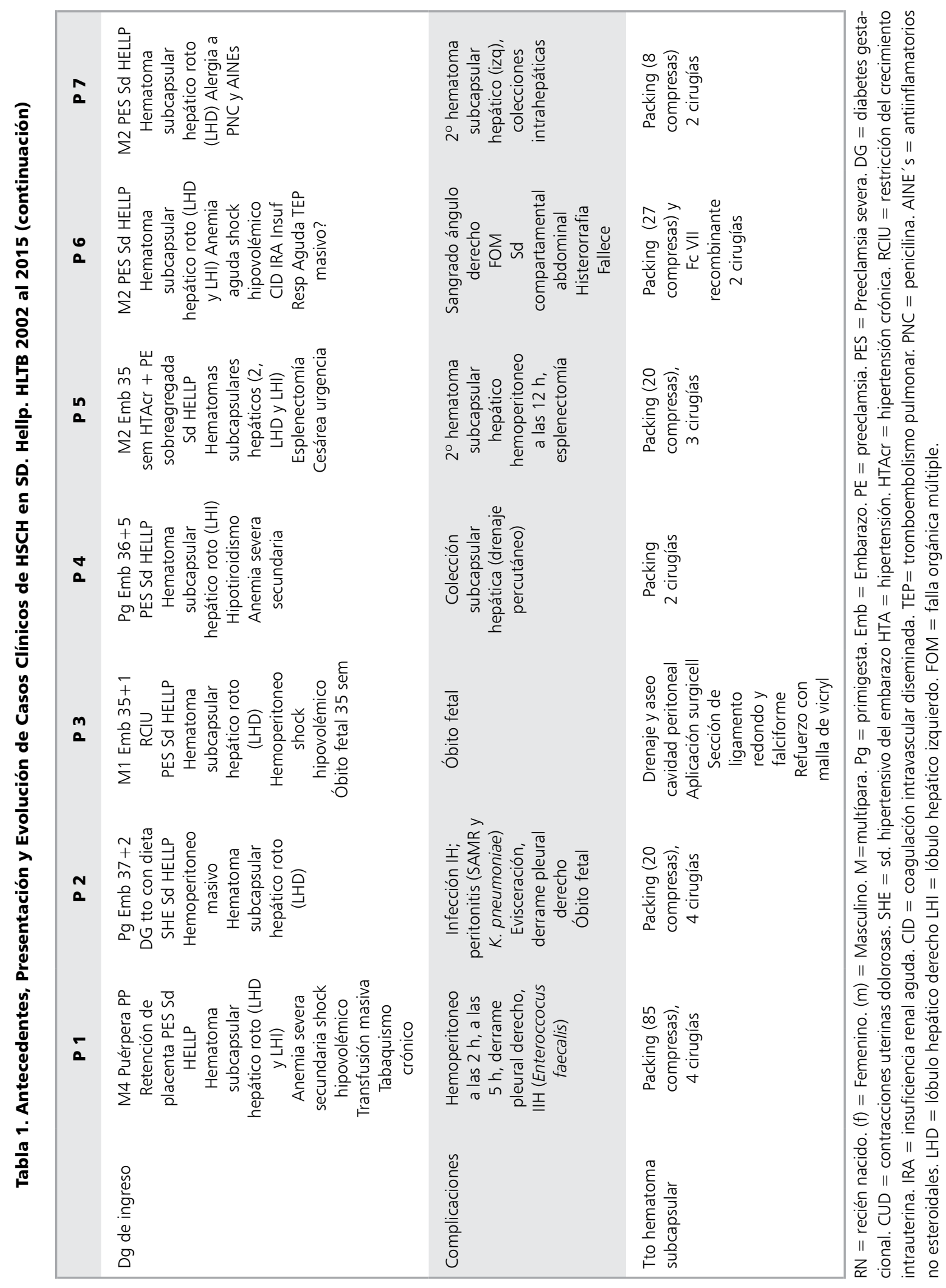




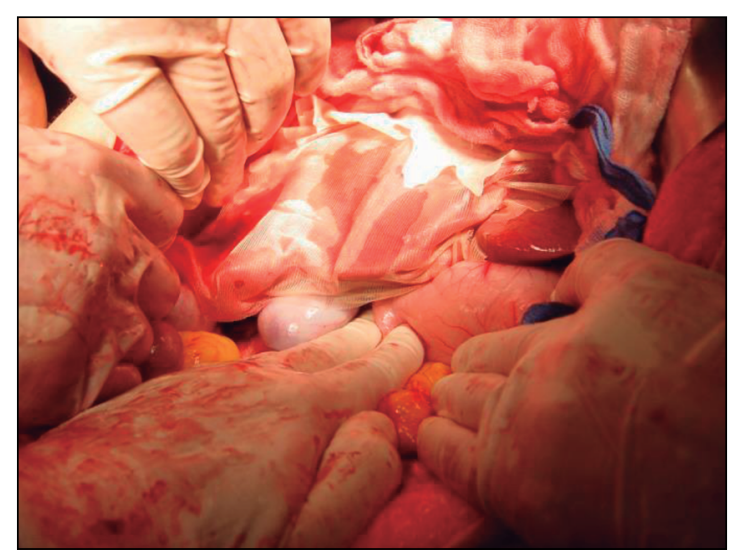

Figura 1. Control hemorragia.

\section{Mortalidad}

De las 7 pacientes que ingresaron con diagnóstico de preeclampsia y síndrome HELLP que se complicaron con la rotura del $\mathrm{HSCH}$, solo una paciente (14\%) falleció, y las otras seis $(86 \%)$ fueron dadas de alta en buenas condiciones generales.

\section{Resultado perinatal}

De los 7 embarazos, hubo 2 óbitos fetales (de $35+1$ y $37+2$ semanas) diagnosticados al ingreso $(29 \%)$, y 5 recién nacidos vivos $(71 \%)$.

Con respecto a las complicaciones, las más frecuentes correspondieron a hemoperitoneo recurrente que requirió reintervención (3 pacientes), infecciones intrahospitalarias (1 por Staphylococcus aureus meticilino resistente (SAMR) y Klebsiella pneumoniae y 1 por Enteroccocus faecalis), derrame pleural (2 pacientes) y evisceración (1 paciente).

En promedio, los días de estadía en UCI fueron 10 , con un rango de 4 a 25 días y una mediana de 6 días.

Cabe destacar el caso de la paciente que falleció ( $\mathrm{N}^{\circ}$ 6), 37 años, multípara de 2, con 32 semanas de embarazo al ingreso, con cifras tensionales elevadas (hasta 179/90 $\mathrm{mmHg}$ ), cefalea, y tinnitus. Proteinuria de $345 \mathrm{mg} / 24 \mathrm{~h}$, sin alteración de plaquetas ni pruebas hepáticas al ingreso. A las 48 h refirió epigastralgia, se objetivó elevación de transaminasas y trombocitopenia leve $(129.000 \mathrm{x}$ $\left.\mathrm{mm}^{3}\right)$ al $5^{\circ}$ día, por lo cual se inició sulfato de magnesio. Se realizó cesárea de urgencia, obteniéndose un recién nacido (RN) masculino, $2.080 \mathrm{~g}$, APGAR 8-8. En el postoperatorio inmediato evolucionó con palidez, epigastralgia y omalgia derecha, hematocrito $27 \%$ y hemoglobinemia de $8,6 \mathrm{~g} / \mathrm{dL}$, plaquetas de $116.000 \mathrm{x} \mathrm{mm}^{3}$. Se decidió reexploración quirúrgica; se realizó una LMSIU y se encontró un extenso hematoma subcapsular hepático bilobar, que se rompió al explorar. Se realizó packing con 17 compresas sobre malla de vicryl y se dejó bolsa de Bogotá, que fue reacomodado 6 h después con 10 compresas más, por persistencia de sangrado a través de herida operatoria, cuando se constató sangrado por ángulo derecho de histerorrafia, por sitio de inserción de catéter venoso central (CVC) y vías venosas, como manifestación clínica de coagulación intravascular diseminada (CID) en curso. Se decidió manejo con factor VII recombinante 4 ampollas, además de $11 \mathrm{U}$ de glóbulos rojos, $23 \mathrm{U}$ de plaquetas, 12 $\mathrm{U}$ de crioprecipitado, y $13 \mathrm{U}$ de PFC. Se mantuvo siempre grave, en falla multiorgánica, requirió hemodiálisis continua en modalidad de hemofiltración de alto volumen (HFAV). Luego de la $4^{\mathrm{a}}$ y última cirugía, evolucionó con shock hipovolémico y cardiogénico, que no mejoró con el uso de drogas vasoactivas ni con HFAV, y con falla respiratoria catastrófica sin respuesta a ventilación mecánica. Se interpretó como tromboembolismo pulmonar masivo secundario a trombosis venosa profunda bilateral, sumada a ectasia profunda del retorno venoso por la compresión de los packing o a trombosis genuina. Falleció al cuarto día después de la cesárea.

\section{Discusión}

El hematoma subcapsular hepático roto es una complicación rara del SHE y del síndrome HELLP, con una incidencia de 1/45.000 a1/250.000 nacidos vivos ${ }^{6}$.

El HSCH se presenta en cerca de 1-2\% de los síndromes HELLP o preeclampsia (PE) ${ }^{5,6,89}$.

Esta complicación suele presentarse entre las 28 y 36 semanas de embarazo ${ }^{20,21}$. Habitualmente ocurre durante el tercer trimestre del embarazo y rara vez en el puerperio ${ }^{22}$, tal como ocurrió en los casos revisados.

Esta revisión, que abarcó 13 años, donde hubo 86.858 partos, se presentaron siete casos de hematoma subcapsular hepático roto en pacientes con síndrome HELLP, con una incidencia de $1 / 12.408$ nacidos vivos, mayor que la reportada en 
la literatura. Destaca solo un fallecimiento, lo cual representa una mortalidad de $14 \%$, cifra muy inferior a la reportada en la literatura $(59 \%-62 \%)^{6,13-17}$.

El diagnóstico del HSCH es difícil de hacer dado que los síntomas son poco específicos. El síntoma predominante en nuestras pacientes fue la epigastralgia, que se presentó en $71 \%$. Este es explicado por la necrosis tisular hepática, oclusión por trombos de fibrina o material hialino en capilares periportales y sinusoides. Por lo anterior, podemos concluir que el dolor es un síntoma constante, que puede presentarse desde días previos, siendo muy importante contar con un alto índice de sospecha clínica para un diagnóstico precoz.

La hipertensión y proteinuria están presentes en aproximadamente $85 \%$ de los casos de las pacientes con síndrome HELLP ${ }^{23,24}$.

Respecto a los parámetros de laboratorio, no existe correlación con la rotura o hematoma hepático, sin embargo, todas las pacientes estudiadas en este período manifestaron elevación importante en las enzimas hepáticas, LDH y trombocitopenia importante.

En cuanto al estudio, frente a una paciente en la segunda mitad del embarazo que presente dolor en el cuadrante abdominal superior, repentino e intenso, asociado a omalgia, cervicalgia e hipotensión, o combinación de estos síntomas, planteando descartar $\mathrm{HSCH}$, resulta de gran utilidad la toma de imágenes como tomografía computada (TAC) o resonancia magnética (RM), siendo clave el alto índice de sospecha para el diagnóstico precoz ante cualquiera de estos síntomas. TAC y RM son más sensibles que la ecografía abdominal, sin embargo, esta última debe ser utilizada ante la falta de los métodos anteriores o gravedad de la paciente.

En el enfrentamiento y manejo de una paciente con HSCH deben distinguirse 3 aspectos fundamentales: La interrupción del embarazo, tratamiento del HSCH y tratamiento del compromiso multisistémico en una unidad de cuidados intensivos. La interrupción del embarazo es el único tratamiento definitivo y con efecto beneficioso para la madre y el feto. La vía sugerida es la cesárea, dado que las contracciones pueden favorecer la rotura del hematoma. Si está establecido el diagnóstico, es preferible una laparotomía media a la incisión de Pfanennstiel, para la mejor visualización de la cavidad abdominal ${ }^{17}$.

Como segundo punto, el tratamiento del $\mathrm{HSCH}$ y su rotura incluye un amplio espectro de conductas terapéuticas. La conducta a seguir dependerá del estado hemodinámico de la paciente y si el hematoma está roto o no.

El tratamiento conservador del hematoma hepático intacto requiere estricta monitorización clínica, de laboratorio e imagenológica. El uso de escleroterapia de arterias hepáticas se perfila con un rol protagónico en el tratamiento de esta complicación, requiriendo como paso previo, el diagnóstico oportuno del hematoma a través de imágenes.

Según Shames BD y col ${ }^{33}$, la cirugía debe reservarse para casos de inestabilidad hemodinámica, rotura con hemoperitoneo, pérdida hemática contínua, aumento del volumen del hematoma e infección del hematoma. Las opciones de tratamiento quirúrgico se basan en la gravedad del daño del órgano y las dividen en 4 grupos:

1. Compresión de la zona hepática sangrante (packing).

2. Interrupción del flujo sanguíneo de la arteria hepática.

3. Resecciones hepáticas parciales.

4. Hepatectomía total con trasplante de hígado.

La compresión del tejido sangrante puede realizarse con compresas, mallas sintéticas(Figura 2), puntos hemostáticos en el parénquima hepático,

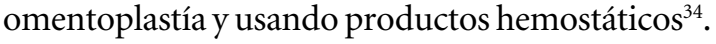
El packing o empaquetamiento con compresas suele ser el tratamiento de entrada (Figura 3). Distintos autores han utilizado esta técnica con relaparotomía para evaluación y retirada de las compresas a las 48 h o antes, según la evolución de la paciente, con la obtención de resultados satisfactorios y buena sobrevida ${ }^{12,17}$. El trasplante hepático es el último recurso a considerar cuando la hemorragia hepática no puede controlarse por los métodos quirúrgicos convencionales o se produce un fallo hepático progresivo secundario a la necrosis hemorrágica extensa, sin embargo, presenta muy alta morbimortalidad ${ }^{32,33}$.

En un tercer punto, como parte del enfrentamiento multisistémico, hay que tener en cuenta que algunas veces, la hemostasia inicial no tiene éxito, dado que el hematoma subcapsular produce una avulsión extensa de la cápsula hepática, sumado a la trombocitopenia preexistente y al trastorno progresivo de la función hepática, con la coagulopatía consecuente. Estudios recientes han mostrado la posibilidad de utilizar factor 


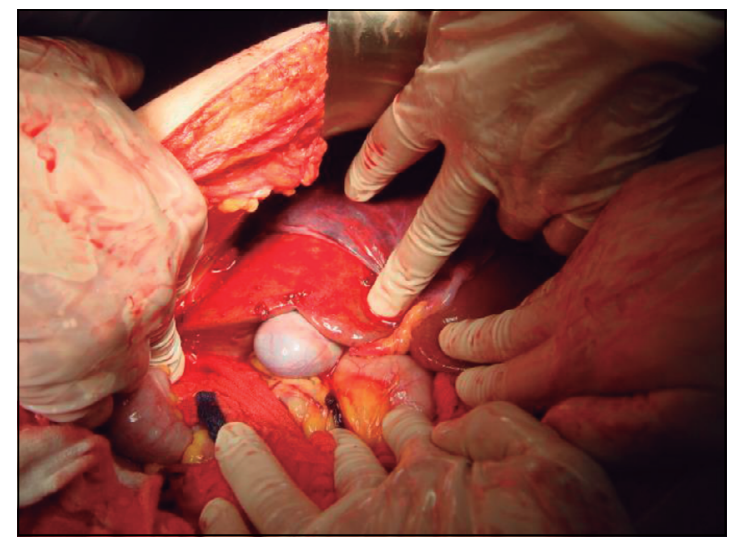

Figura 2. Control hemorragia.

VIIa recombinante como alternativa terapéutica, al estimular la formación de tapones hemostáticos en los sitios de hemorragia. No se ha utilizado en forma extensa debido al riesgo eventual de eventos trombóticos y embolía pulmonar, junto con el riesgo asociado de coagulación intravascular diseminada que posee el síndrome HELLP y que teóricamente este agente podría exacerbar.

\section{Conclusiones}

El hematoma subcapsular hepático roto asociado a síndrome HELLP es una complicación rara y muy grave del síndrome hipertensivo del embarazo, que requiere un alto índice de sospecha para un diagnóstico oportuno y tratamiento quirúrgico precoz y multidisciplinario, lo que es fundamental en el pronóstico del cuadro.

Se recomienda el uso de imágenes, con TAC o en su defecto una ecografía abdominal como estudio confirmatorio ante la sospecha clínica de HSCH secundario a HELLP.

Según el estado hemodinámico de la paciente, tamaño del hematoma, indemnidad de la cápsula, se debe ofrecer la intervención. Se podría plantear un manejo expectante si el hematoma es pequeño, no hay progresión del tamaño y hay estabilidad hemodinámica. La escleroterapia de arterias hepáticas se debe plantear igualmente cuando la paciente se encuentra estable hemodinamicamente y con una cápsula de Glisson indemne.

El manejo quirúrgico se indica en una paciente hemodinamicamente inestable o con evidencia di-

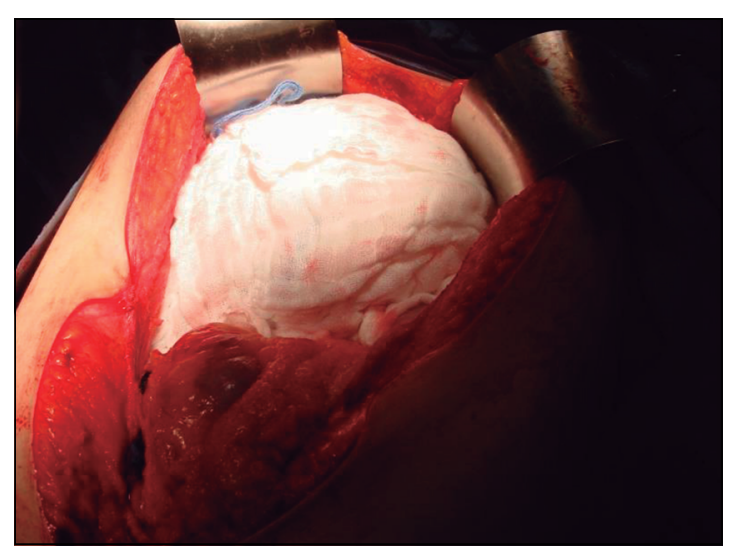

Figura 3. Packing final.

recta (visualización intraoperatoria o imágenes) de la rotura del HSCH o su inminente compromiso. Dentro de las técnicas más reconocidas en nuestro medio y en nuestro servicio, el packing hepático ha demostrado ser la primera indicación para contener la hemorragia y permitir una estabilización sistémica con menor morbimortalidad.

A través de estos siete casos de HSCH que ocurrieron en estos 13 años de experiencia en nuestro servicio, se presenta una actualización en el tema, que nos guiará frente a un nuevo episodio.

El manejo de estas pacientes de extrema gravedad debe ser realizado en centros de alta complejidad, con equipos multidisciplinarios médico-quirúrgicos, que cuenten con unidades de cuidados intensivos, neonatología e, idealmente, con radiología intervencionista, para su correcta monitorización, soporte y tratamiento. En su defecto, frente a la sospecha, estas pacientes deben ser derivadas a su centro de referencia más cercano que cuente con los servicios anteriormente nombrados, dada la heterogeneidad de equipamiento y complejidad de las maternidades a lo largo de nuestro país.

\section{Referencias}

1. Stone JH, HELLP syndrome: Hemolysis, elevated liver enzymes, and low platelets. JAMA 1998; 280: 559-62.

2. Sibai BM, Taslimi MM, el-Nazer A, Amon E, Mabie BC, Ryan GM. Maternal-perinatal outcome associated with the syndrome of hemolysis, elevated liver enzymes, and low platelets in severe preeclampsia-eclampsia. Am J Obstet Gynecol 1986; 155: 501. 
3. Sibai BM. The HELLP syndrome (hemolysis, elevated liver enzymes, and low platelets): much ado about nothing? Am J Obstet Gynecol 1990; 162 (2): 311-6.

4. Barton JR, Sibai BM. Hepatic imaging in HELLP syndrome. Am J Obstet Gynecol 1996; 174: 1820-7.

5. Nunes JO, Turner MA, Fulcher AS. Abdominal imaging features of HELLP syndrome: a 10-year retrospective review. AJR Am J Roentgenol 2005; 185 (5): 1205-10.

6. Sherbahn R. Spontaneous ruptured subcapsular liver hematoma associated with pregnancy. A case report. J Reprod Med 1996; 41: 125-8.

7. Nelson DB, Dearmon V, Nelson MD. Spontaneous rupture of the liver during pregnancy: a case report. J Obstet Gynecol Neonatal Nurs 1989; 18: 106-13.

8. van Dyke RW. "The liver in pregnancy” in: Zakim D and Boyer T, editors, Hepatology: A Text Book of Liver Disease, pp. 1408-18, 2nd edition. W.B. Saunders Company, Philadelphia, USA. 1990.

9. Westengaard L. Spontaneous rupture of the liver in pregnancy. Acta Obstet Gynecology 1983; 59: 559-61.

10. Stevenson JT, Graham DJ. Hepatic hemorrhage and the HELLP syndrome: a surgeon's perspective. Am Surg 1995; 61: 756-60.

11. Perucca E, Domínguez C, González D, Ricci P, Estay R, Siebert A, Iracheta A. Rotura Hepática Espontánea En Síndrome HELLP. Rev Chil Obstet Ginecol 2003; 68 (1): 7-12.

12. Vera E, Pérez A, Lattus J, Barrera V, Campaña G, Kat$\tan$ MJ, et al. Rotura hepática asociada a preeclampsia severa y síndrome HELLP: manejo y tratamiento con taponamiento intraabdominal temporal de compresas. Rev Chil Obstet Ginecol 2004; 69 (4): 319-27.

13. Greenstein D, Henderson JM, Boyer TD. Liver hemorrhage: recurrent episodes during pregnancy complicated by preeclampsia. Gastroenterology 1994; 106: 1668-71.

14. Sibai BM. Pregnancies complicated by HELLP Syndrome. Subsequent pregnancy outcome and long termprognosis. Am J Obstet Gynecol 1995; 172: 125-9.

15. Rath W, Loos W, Kuhn W. HELLP syndrome, Zentralbl Gynakol 1994; 116: 195-201.

16. Reck T, Bussenius-Kammeree M, Ott R, Müller E, Beinder E, Hohenberger W. Surgical treatment of HELLP syndrome asociasted liver ruptura an update. Eur J Obst y Ginecol 2001; 99: 57-65.

17. Pérez AC, Martínez WM, Hoefler SS, Mollo EA, Campaña GV, Czwiklitzer GS. Hematoma subcapsular hepático roto asociado a pre-eclampsia severa y síndrome HELLP como urgencia quirúrgica. Técnica quirúrgica. Rev Chil Cir 2007; 59 (6): 403-7.

18. Weinstein L. Syndrome of hemolysis, elevated liver enzymes and low platelet count: a severe conse-quence of hypertension in pregnancy. Am J Obstet Gynecol 1982; 142: 159-67.

19. Norwitz ER, Hsu CD, Repke JT. Acute complications of preeclampsia. Clin Obstet Gynecol 2002; 45: 308-29.

20. Marsh FA, Kaufmann SJ, Bhabra K. Surviving hepatic rupture in pregnancy-a literature review with an illustrative case report. J Obstet Gynaecol 2003; 23: 109-13.

21. Sibai BM, Ramadan MK, Usta I, Salama M, Mercer BM, Friedman SA. Maternal morbidity and mortality in 442 pregnancies with hemolysis, elevated liver enzymes, and low platelets (HELLP syndrome). Am J Obstet Gynecol 1993; 169: 1000.

22. Richard HW, Barry MS. Postpartum rupture of a subcapsular hematoma of the liver. Acta Obstet Gynecol Scand 1992; 71: 394-7.

23. Sibai BM. Diagnosis, controversies and management of the syndrome of hemolysis, elevated liver enzymes and low platelet count. Obstet Gynecol 2004; 103: 981.

24. Schwartz ML, Lien JM. Spontaneous liver hematoma in pregnancy not clearly associated with preeclampsia: a case presentation and literature review. Am J Obstet Gynecol 1997; 176: 1328.

25. Grand'Maison S, Sauvé N, Weber F, Dagenais M, Durand M, Mahone M. Hepatic rupture in hemolysis, elevated liver enzymes, low platelets syndrome. Obstet Gynecol 2012; 119: 617.

26. Rinehart BK, Terrone DA, Magann EF, Martin RW, May WL, Martin JN Jr. Preeclampsia-associated hepatic hemorrhage and rupture: mode of management related to maternal and perinatal outcome. Obstet Gynecol Surv 1999; 54: 196-202.

27. Araujo AC, Leao MD, Nobrega MH, Bezerra PF, Pereira FV, Dantas EM, et al. Characteristics and treatment of hepatic rupture caused by HELLP syndrome. Am J Obstet Gynecol 2006; 195: 129-33.

28. Kozar RA, Moore FA, Moore EE, West M, Cocanour CS, Davis J, et al. Western Trauma Association critical decisions in trauma: nonoperative management of adult blunt hepatic trauma. J Trauma 2009; 67: 1144-8; discussion 1148-9.

29. Henny CP, Lim AE, Brunmmelkamp WH, Buller HR, Cate JWT. A review of the importance of acute multidisciplinary treatment following spontaneous rupture of liver capsule during pregnancy. Surg Gynecol Obstet 1983; 156: 593-8.

30. Woodhouse DR. Conservative management of spontaneousrupture of liver in pregnancy. Case report. British J Obstet Gynecol 1986; 93: 1097-9.

31. Mihu D, Costin N, Mihu CM, Seicean, Ciortea R. HELLP Syndrome - a Multisystemic Disorder. J Gastrointestin Liver Dis 2007; 16 (4): 419-24. 
32. Pérez MT, Sáez V, Hematoma Subcapsular Hepático. Grave Complicación del Embarazo, Rev Elec de las Ciencias en Cienfuegos. Medisur 2010; 8 (6): 445-51.

33. Shames BD, Fernández LA, Sollinger HW, Chin LT, D’Alessandro AM, Knechtle J, et al. Liver Transplantation for HELLP Syndrome. Liver Transpl 2005; 11 (2): 224-8.

34. Shrivastava VK, Imagawa D, Wing DA. Argon Beam Coagulator for Treatment of Hepatic Rupture With Hemolysis, Elevated Liver Enzymes, Low Platelets (HELLP) Syndrome. Obstet Gynecol 2006; 107 (2 part 2): 525-6.

35. Butte JM, Jarufe CN, Vuletin SF, Martínez CJ. Tratamiento con malla de poliglactina del hematoma subcapsular hepático roto. Rev Chil Cir 2006; 58 (5): 377-81.

36. Reck T, Bussenius-Kammerer M, Ott R, Müller V, Beinder E, Hohenberger W. Tratamiento quirúrgico de la rotura hepática asociada con el síndrome HELLP-actualización. European Journal of Obstetrics \& Gynecology and Reproductive Biology (Ed. Española) 2002; 2: 24-32.

37. Hedner U, Erhardsten E: Potential role of recombinant factor VIIa in transfusion medicine. Transfusion 2002; 42: 114-24.

38. Merchant SH, Mathew P, Vanderjagt TJ, Howdieshell TR, Crookston KP. Recombinant factor VIIa in management of spontaneous subcapsular liver hematoma associated with pregnancy. Am J Obstet Gynecol 2005; 103: 1055-8.
39. Guntupalli S, Steingrub J, Hepatic disease and pregnancy: An overview of diagnosis and management. Crit Care Med 2005; 33 (10): 332-9.

40. Goel A, Ramakrishna B, Zachariah U, Ramachandran J, Eapen CE, Kurian G, et al. How accurate are the Swansea criteria to diagnose acute fatty liver of pregnancy in predicting hepatic microvesicular steatosis? Gut 2011; 60: 138 .

41. Minakami H, Morikawa M, Yamada T, Yamada T, Akaishi R, Nishida R. Differentiation of acute fatty liver of pregnancy from syndorome of hemolysis, elevated liver enzymes and low platelet counts. J Obstert Gynaecol Res 2014; 40: 641.

42. Stella CL, Dacus J, Guzmán E, Dhillon P, Coppage K, How $\mathrm{H}$, et al. The diagnostic dilemma of thrombotic thrombocytopenic purpura/hemolytic uremic syndrome in the onstetric triage and emergency deparament: lessones from 4 tertiary hospitals. Am J Obstet Gynecol 2009; 200: 381.e1.

43. Martin JN Jr, Bailey AP, Rehberg JF, Owens MT, Keiser SD, May WL. Thrombotic thrombocytopenic purpura in 166 pregnancies: 1955. 2006. Am J Obstet Gynecol 2008; 199: 98.

44. Van de Minkelis JL, Steenvoorde P, Baranski, A, Liver Rupture in a Patient with HELLP Syndrome Successfully Treated with Extensive Surgery Combined with Recombinant Factor VIIa. Actachirbelg 2006; 106: 602-4. 